\title{
Design of Coal Mine Underground Drainage Pump Monitoring and Controlling System using ARM7 and Touch Screen
}

\author{
Devashish Saha \\ ECE Department, \\ Lingayas University, \\ Faridabad.
}

\author{
S.V.A.V. Prasad,Ph.D \\ Dean \\ Lingaya's University \\ Faridabad.
}

\author{
Nitin Saluja \\ Assistant Professor, \\ Lingayas University, \\ Faridabad.
}

\begin{abstract}
This paper is based on designing a low cost coal mine underground drainage pump monitoring and controlling system based on 32-bit microcontroller i.e. ARM7 and touch screen. By using a touch screen device the human machine interface will become more user- friendly. A graphical LCD will be used to display the parameters on the screen which can be analysed and accordingly controlling actions can be performed. Internal ADC will be used to implement the sensors as well as touch screen panels.
\end{abstract}

\section{Keywords}

Underground drainage system; GLCD; ARM7; LPC2148; ADC; LCD

\section{INTRODUCTION}

Coal mine production need an equipment for its safety i.e. able to drain out all inflows during coal production as well as handle production situations, hence a coal mine underground drainage system is very important equipment. Safe, efficient and economical control over the operation of underground drainage system is one of the effective ways to reduce the production costs of coal mine and is of great significance to safe production in coal mine [1]. This project is based on designing a very low cost touch screen device for coal mine underground drainage pump monitoring and controlling system based on 32-bit controller i.e. ARM7 and touch screen. Taking advantage of touch screen interface to display and control related parameters and dynamic enhances the considerable and operability [2]. With recent proliferation of touchscreen-equipped mobile devices typified by iPhones and Android phones, there has been rapid growth of interest in touch sensing techniques that enable a number of novel user interface (UI) possibilities, e.g., gestural interactions [10]. By using a touch screen device the human machine interface will become straightly [4], more user friendly and can be very easily used and control the overall situations in production system. Because of its durability, fast responsible, easy to communication, abundant performance ability, flexible [5] and easy access and space saving, the touch-screen has generally replaced the mouse and keyboard, and become the primary input device of embedded systems [3] [6]. ARM7 controller will be used for designing this device since it is a low power device and it has an on-chip ADC so an external
ADC IC is not required. This will help in keeping the cost of the design considerably low. A graphical LCD will be used to display the parameters on the screen which can be analysed and accordingly controlling actions can be performed. Internal ADC will be used to implement the sensors as well as touch screen panels. In this project a 4-wire touch screen has been used which will use 2 channels of internal ADC.

The main goal of the design should be to keep the cost of the system low and compare the cost with the existing system implemented using PLC. At last all parameters of different type of touch screen are compared and finally selecting the appropriate touch screen for the device we have implemented this system. Finally a complete working prototype of the device will be designed and implemented using ARM7 controller having a touch screen interface for user interaction.

\section{COMPARISION WITH EXISTING SYSTEM}

Existing system rely on bulky hardware technology that consists of PLC, two 12-bit 4-channel ADC modules and 10-inch touch screen [1]. This system is based on ARM7 microcontroller to design the complete system which is based on LPC2148 member of the given family, The ARM7 is a general purpose 32-bit microprocessor, which offers high performance and very low power consumption. The ARM architecture is based on Reduced Instruction Set Computer (RISC) principles and the instruction set is simple [11]. The related decode mechanism are much simpler than those of micro programmed Complex Instruction Set Computers. This simplicity results in a high instruction throughput and impressive real-time interrupt makes it ideally suited to high-volume applications with memory restrictions, or applications where code density is an issue [11]. A 4wire touch screen which is also easy to interface with microcontroller as it will give the analog voltages according to the touched region and LPC 2148 has an on chip ADC module so we don't need an external ADC module to convert analog data into digital data. Pump driver will provide the necessary driving circuit to drive the pump which we will be using to pump the underground drainage from the coal mine. With the help of level sensors the accurate information will be gathered about the level of water in the underground drainage system and accordingly pumping action can be performed. 


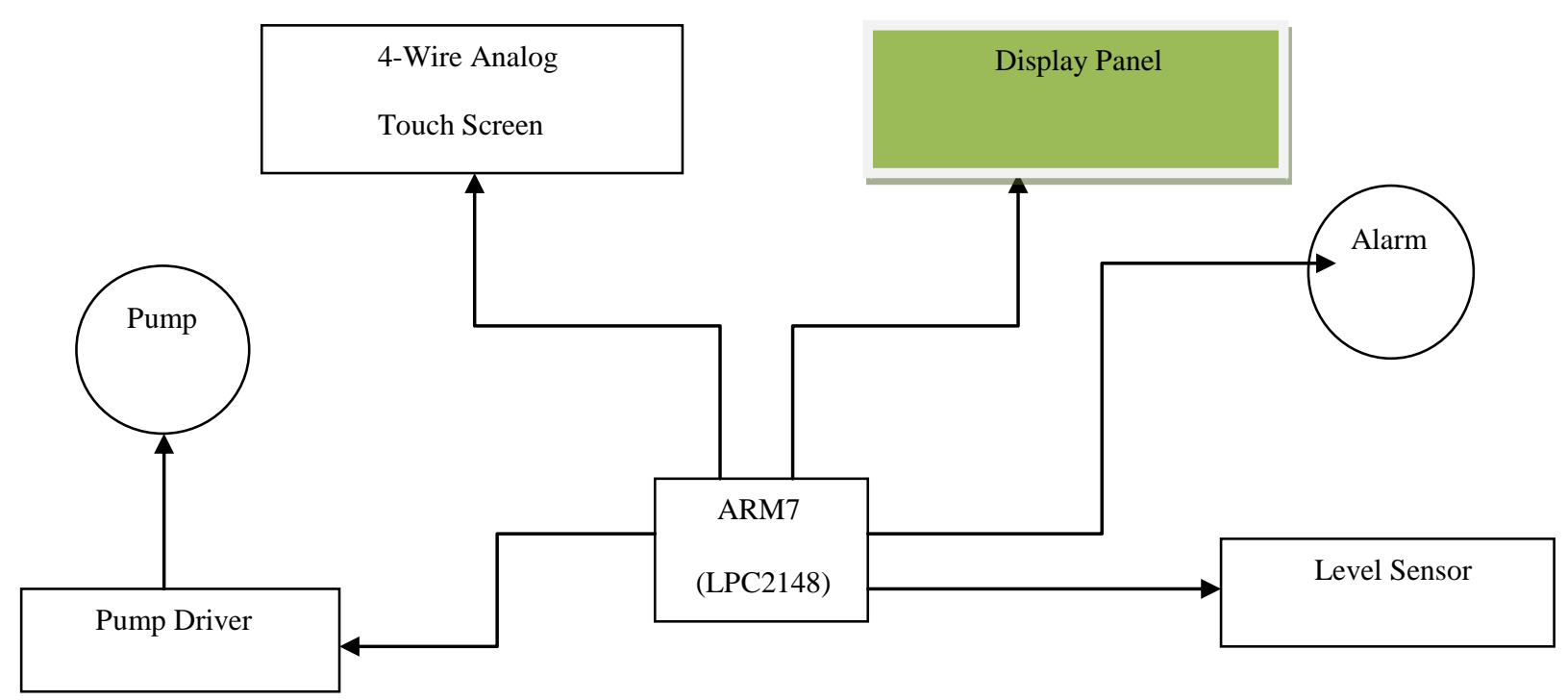

Figure 1: Hardware Architecture

\section{LIMITATIONS OF EXISTING SYSTEM}

Although existing system is based on advanced technology such as PLC and 10-inch touch panel human computer interface but system is too bulky, heavy and very complex as external ADC's are used. The overall cost of the system is also much as PLC's are very costly. However the proposed design is based on ARM family of microcontroller and is a low power, compact and low cost device. The main advantage of the new proposed design is that it has on chip support for analog to digital converter so there is no need to use external hardware chips for these.

\section{HARDWARE ARCHITECTIRE OF ARM7 ENABLED SYSTEM}

ARM7 microcontroller has been used to design the complete system which is based on LPC2148 member of the given family. A graphical display panel is used to design a display unit which will give details of the controls which has to be performed for the Design of Coal Mine Underground Drainage Pump Monitoring and Controlling System. The display panel will be interfaced with ARM7 micro-controller. The interfacing connections will have data bus and control bus of GLCD to be connected with the micro-controller. The data bus will be used to send data to the display panel and the control bus will be used to send and receive commands to and from the display panel. Multiple options are there for touch screen which can be used for this design but we have used a 4 wire analog touch screen which keeps the cost of the system low. The 4 wire interface is also easy to interface with microcontroller as it will give the analog voltages according to the touched region and LPC2148 has an on chip ADC module so we don't need an external ADC module to convert analog data into digital data. Pump driver will provide the necessary driving circuit to drive the pump which we will be using to pump the underground drainage from the coal mine. With the help of level sensors the accurate information will be gathered about the level of water in the underground drainage system and then necessary pumping operations can be performed.

\section{TOUCH SCREEN USER INTERFACE}

Touch sensors are applied to simplify the humanmachine interfacing [9] [7]. Touch-screen all-in-onemachine is the simplest, most convenient and most natural human-computer interaction method as a new kind of computer input devices [4]. The touch screens are capable of multi touch interaction [8]. The above display is a graphical LCD display having 128 X64 pixels configuration. This display is used to design user interface which will help the user to select the desired operation which he has to perform using the coal mine drainage system.

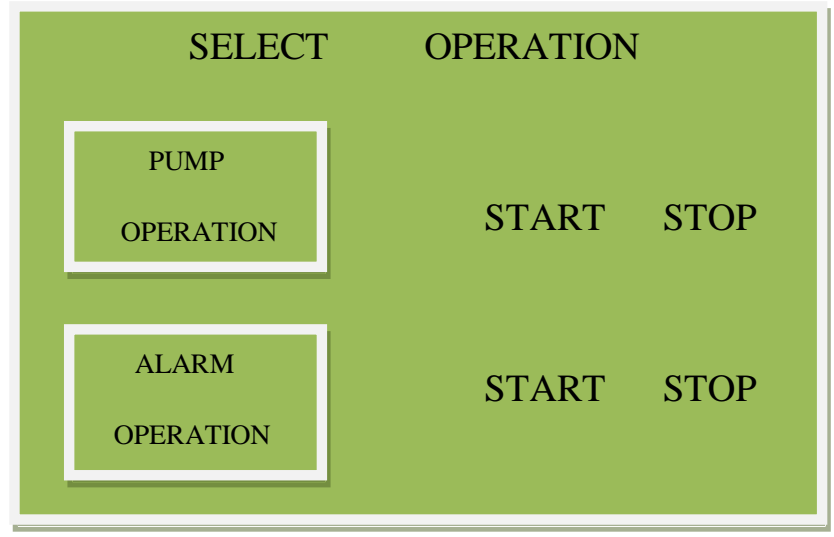

Figure 2:Touch Screen Based Graphical LCD Display Panel 
The page which displays the operation related to pump and alarm is shown above. The pump and alarm operation can be selected using start and stop switch which are displayed on the right side of the screen. The operation will be selected by giving inputs from the touch screen which is mounted on the surface of the graphical LCD. According to the $\mathrm{x}$ and $\mathrm{y}$ coordinates touched on the touch screen the desired operation is performed.

\section{OPERATIONS SELECTION IN CASE OF HAZARDOUS SITUATIONS Operation selection from} the touch panel will be done based on operating mode of the system. We have two operating modes in this system viz. active mode and inactive mode. The change in mode can be done just by a single touch on the display panel and inactive mode will be toggled to the active mode. When the system is running in the active mode then all the contact sensors will become active and can sense the level of water in the underground drainage. According to the level of water risen in the drainage system the alert message or indication can be generated on the buzzer. Apart from the alert signal generated on the alarm the pumping operation can be started or stopped according to the different level of water in the drainage. Hence multiple operations can be selected.

\section{PUMP AND CONTROL ACTIONS}

\subsection{Pumping Operation}

In case of hazardous situation i.e., in case water level increases above mentioned level or dangerous level, by using touch panel the user can start the pump. The embedded system sends command to the pump to start and in this way the pump starts draining out the water up to the normal level.

\subsubsection{Scheduled Pumping Operation}

The pump starts automatically as per scheduled time i.e., in areas where water keeps on increasing, the pump starts and drains out the water automatically no need to start or stop the pump manually.

\subsubsection{Pump Cut-Off}

The pump starts draining out water and when water reached at normal level, the user can stop the pump with the help of touch panel. The embedded system sends command to the pump to stop.

\subsection{Alert Message Using Alarm}

If a situation comes that water is reaching at marked dangerous level the alarm gets start and giving beep sound. The alert message helps the people in coal mine to understand the situation of underground water level.

\subsection{Warning Message}

If the situation comes that water reached at dangerous level, the warning message from alarm help the people in coal mine to understand that the coal mine production should be stop immediately and vacate the coal mine.

\section{ADVANTAGES OF NEW DESIGNED SYSTEM}

ARM7 microcontroller is used to design the complete system which is based on LPC2148 member of the given family. This member of ARM7 micro-controller family is very cheap and is easily available in the market. On the other hand the PLC modules which are available in the market are expensive as compared to this controller. Another advantage of this system is that it does not require external convertor circuits which will convert analogue signal into digital signal since it has internal support of ADCs available inside LPC2148 controller. The ARM architecture is based on Reduced Instruction Set Computer (RISC) principles and it is very easy to program it in embedded $\mathrm{C}$ language as compared to programming o PLC. Touch screen panel will make this new system user friendly and more precise in selecting inputs. The overall cost of the system becomes less as it has lot of on chip support and can be run on batteries. Touch screen driver circuit and pump driver circuit are compact in size and will provide the necessary driving functionality to drive them.

\section{RESULT}

Under practical conditions the desired operations are performed using this device and the complete underground drainage system is managed. Alarm operation when required is executed and alert signals are generated using same alarm. Pumping operation is also performed in two ways i.e. manual and automatic which will result in cleaning of the underground drainage.

Following table gives the results of the different features implemented in the designed system.

\begin{tabular}{|c|l|l|}
\hline S. No. & \multicolumn{1}{|c|}{ Feature } & Characteristics \\
\hline 1. & Response time of touch screen & $40 \mu \mathrm{sec}$ \\
\hline $\mathbf{2 .}$ & Alert signal duration for level 1 & $1 \mathrm{sec}$. \\
\hline $\mathbf{3 .}$ & Alert signal duration for level 2 & $0.5 \mathrm{sec}$ \\
\hline $\mathbf{4 .}$ & Alert signal duration for level 3 & $0.25 \mathrm{sec}$ \\
\hline $\mathbf{5 .}$ & Alert signal generation delay & $5 \mu \mathrm{sec}$ \\
\hline
\end{tabular}

\section{CONCLUSION}

In conclusions, a complete low cost hand held embedded system has been designed which is having a touch screen user interface based of ARM7 microcontroller for underground coal mine drainage pump monitoring and controlling system. It has an in built ADC unit which help to reduce the cost of the entire system since supporting hardware required for the system has also been reduced. Replacing PLC by ARM7, the cost of the system is further reduced drastically. By adding various sensors it could be possible to enhance the system capabilities to provide best working conditions fit for underground mining. With same alarm the alert signals could be generated for various sensors indicating hazardous situations important for mining. Also same pump could do pumping operation of various sites located in the same mine. 


\section{ACKNOWLEDGEMENT}

The authors would like to acknowledge the guidance of faculty of electronics department and all the team members for their support. Our special thanks to Lingayas University for their support and also providing labs with all the required equipments.

\section{REFERENCES}

[1] WU Jing and CHEN Guo jie., "Design of Coal Mine Underground Drainage Pump Monitoring and Controlling System Based on PLC and Touch Screen ", 2011 International Conference on Mechatronic Science, Electric Engineering and Computer, pp.1245-1247, August 19-22, 2011, Jilin, China.

[2] Lijun Fu, Zhenhai Qian, Yan Tang, Meichen Zhu and Hongbin Liu., "Touch screen-based motor bearing fault diagnosis”, 2012 24th Chinese Control and Decision Conference (CCDC),pp22752280,2012

[3] Shuangye Chen and Xiaoyu Qin., "The Research of Touch-screen Calibration Algorithm and Its Application to the Embedded System", 2012 International Conference on Systems and Informatics (ICSAI 2012), pp.483-486, 2012

[4] Zhang Yaojun, Rong Li and Wu Haiyan., "TouchScreen Terminal Application for Remote Data based on Authorized Access", IEEE, pp-218-221, 2011.
[5] Jidapa Rattarojpan and Sumet Umchid., "Design and Development of Touch Screen Based Continuous Passive Motion Device for Knee Rehabilitation", The 2011 Biomedical Engineering International Conference (BMEiCON-2011), pp.237-241, 2011.

[6] Wang Huiqiang, Xing Yanqiu and Xiao Tao., "The Control System of Belt Grinder Which Based On The Touch Screen And PLC", 20IO International Conference on Computer Application and System Modeling (ICCASM 2010), vol-14, pp.438-441, 2010.

[7] Jehyun Jung, Egyul Kim, SeongHun Lee and Jin Hyung Kim., "Scene Text Separation using Touch Screen Interface”, 2009 IEEE, pp.1-5, 2009.

[8] Lepicard Guillaume and Vigouroux Nadine., "Influence of age and interaction complexity on touch screen", 2010 IEEE

[9] R.N. Aguilar and G.C.M. Meijer, "Fast Interface Electronics for a Resistive Touch-Screen”, 2002 IEEE, pp-1360-1363, 2002.

[10] Yongsuk Park, Member, IEEE, Jinwoong Bae, Eungsoo Kim, and Taejoon Park, Member, IEEE, "Maximizing Responsiveness of Touch Sensing via Charge Multiplexing in Touchscreen Devices", IEEE Transactions on Consumer Electronics, vol. 56, no. 3, pp,1905-1910, August 2010.

[11] ARM7 User Manual. 